\title{
Electromagnetics
}

\section{Modeling of Spirals with Equal Dielectric, Magnetic, and Chiral Susceptibilities}

\section{E. Saenz , I. Semchenko , S. Khakhomov , K. Guven , R. Gonzalo , E. Ozbay \& S. Tretyakov}

To cite this article: E. Saenz , I. Semchenko, S. Khakhomov , K. Guven , R. Gonzalo, E. Ozbay \& S. Tretyakov (2008) Modeling of Spirals with Equal Dielectric, Magnetic, and Chiral Susceptibilities, Electromagnetics, 28:7, 476-493, DOI: 10.1080/02726340802322528

To link to this article: http://dx.doi.org/10.1080/02726340802322528

曲 Published online: 15 Sep 2008.

Submit your article to this journal $\asymp$

Џلll Article views: 127

Q View related articles $\sqsubset$

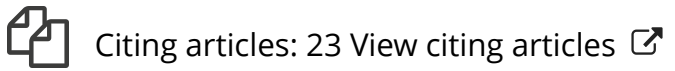




\title{
Modeling of Spirals with Equal Dielectric, Magnetic, and Chiral Susceptibilities
}

\author{
E. SAENZ, ${ }^{1}$ I. SEMCHENKO, ${ }^{2}$ S. KHAKHOMOV, ${ }^{2}$ \\ K. GUVEN, ${ }^{3}$ R. GONZALO, ${ }^{1}$ E. OZBAY, ${ }^{3}$ AND \\ S. TRETYAKOV ${ }^{4}$ \\ ${ }^{1}$ Electrical and Electronic Engineering Department, Public University of \\ Navarra, Spain \\ ${ }^{2}$ Department of General Physicis, Gomel State University, Belarus \\ ${ }^{3}$ Nanotechnology Research Center-NANOTAM, Department of Physics and \\ Department of Electrical and Electronics Engineering, Bilkent University, \\ Turkey \\ ${ }^{4}$ RadioLaboratory, Helsinki University of Technology, Finland
}

\begin{abstract}
In this article, we study spiral particles with optimized design parameters, which can make possible the realization of media with equal dielectric, magnetic, and chiral susceptibilities. Two different spiral structures are investigated: the canonical spiral (which consists of a split loop with two straight-wire sections, orthogonal to the loop plane and connected to the edges of the gap) and the true helix (which is obtained by bending a wire with a constant pitch angle). The transmission and reflection coefficients of arrays of spiral particles are obtained under plane wave excitation by numerical simulation. The properties of slabs formed by periodic chiral and racemic arrays of spirals are investigated. Good agreement is found between the presented results and the previously reported theoretical and experimental studies.
\end{abstract}

Keywords spiral, susceptibility, transmission, reflection

\section{Introduction}

In the last decade, many published articles were devoted to the investigation of the electromagnetic properties of spirals as an element of nonreflecting coverings (Priou et al., 1997; Zoudi et al., 2002; Whites \& Chang, 1997; Tretyakov \& Sochava, 1993; Semchenko et al., 1998; Tretyakov et al., 1996; Cloete et al., 1999; Bohren et al., 1992). Chiral anti-reflection covers were compared with other types of absorbers, and the role of chirality in comparison with the dielectric, magnetic, and absorbing properties was investigated, leading to controversial opinions on the necessity of chirality (Tretyakov et al., 1996; Cloete et al., 1999; Bohren et al., 1992).

At present, the emergence of electromagnetic metamaterials considerably motivates new research on spiral structures. In particular, the so-called "optimal spirals," which offer

Received 2 April 2008; accepted 22 May 2008.

Address correspondence to Elena Saenz, Electrical and Electronic Engineering Department, Public University of Navarra, Campus Arrosadia, Pamplona, Navarra, E-31006, Spain. E-mail: elena.saenz@unavarra.es 
equivalent dielectric, magnetic, and chiral properties (equal permittivity, permeability, and chirality parameter), are of interest. Such materials were proposed, probably for the first time, in Tretyakov et al. (2002) (see also Tretyakov [2003]) as a possibility to realize negative-index media with equal permittivity and permeability. For one particle, the equality of three susceptibilities leads to a wave radiated by the spiral that is circularly polarized in the orthogonal direction to the axis of the spiral (Semchenko et al., 2004, 2007a, 2007b, 2007c, 2007d; Khakhomov et al., 2006; Samofalov \& Tretyakov, 2006). The circularly polarized wave can be radiated for any polarization of the incident wave. Another consequence is that an optimal spiral with particular helicity is completely transparent for circular electromagnetic waves of the same helicity.

When optimal spirals of opposite helicity are paired (i.e., right-handed (RH) and left-handed (LH) in pair), the chiral properties are compensated. The resulting composite with equal numbers of LH and RH optimal spirals exhibits equal dielectric and magnetic responses, which can be utilized to realize metamaterials with unit impedance, i.e., matched to free space (Tretyakov et al., 2002; Tretyakov, 2003). In addition, optimal spirals can be used to create metamaterials with negative refraction of electromagnetic waves (Tretyakov et al., 2002; Tretyakov, 2003; Semchenko et al., 2007e).

In this article, we model and study optimal spirals with equal dielectric, magnetic, and chiral properties theoretically and using numerical simulations.

The article is organized as follows. In Section 2, the two spiral designs (canonical and true spiral) and their geometrical parameters are introduced. The theoretical formulation of the electromagnetic properties of spirals is given in Section 3. Section 4 discusses the optimum canonical and true spirals and their transmission and reflection spectra. The electromagnetic properties of optimal paired spirals is presented in Section 5. The last section concludes the article.

\section{Geometry of the Canonical and True Spirals}

In this work, the scattering properties of two spiral structures are investigated. The first one is the so-called canonical spiral, which consists of a split loop with two wire sections at the edges of the gap, normal to the loop (see Figure 1). The second one is the conventional helix, which is obtained by bending a wire with a constant pitch angle one turn or two turns (see Figure 2). By properly choosing the dimensions of the spirals, it is possible to have equal dielectric, magnetic, and magnetoelectric polarizabilities.

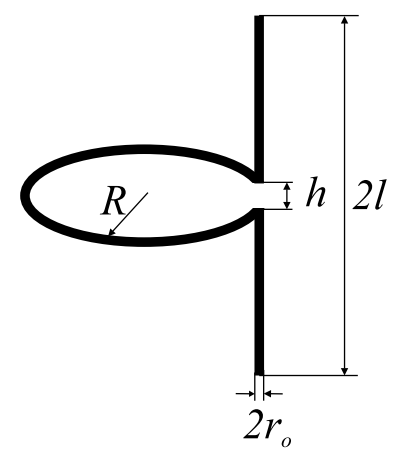

Figure 1. Canonical LH spiral, where $R$ is the radius of the loop, $l$ is the length of the arms, $r_{0}$ is the radius of the wire, and $h$ is the helix pitch. 


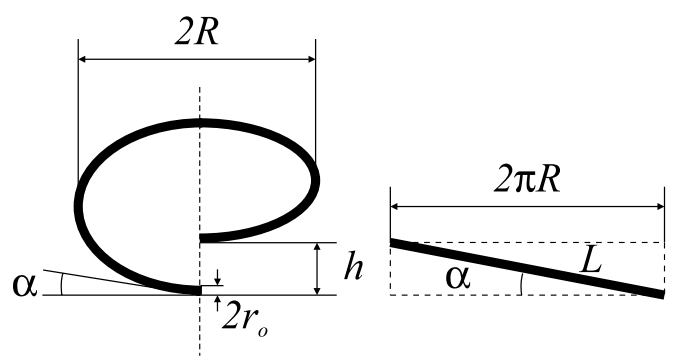

Figure 2. Real one-turn LH spiral, where $N_{c}$ is the number of turns, $R$ is the radius of the turn, $r_{0}$ is the radius of the wire, $\alpha$ is the pitch angle, $h$ is the helix pitch, and $L$ is the total wire length.

Properties of artificial materials with such optimal helical inclusions will be studied in order to realize a chiral material in which two eigenmodes have effective refraction indices equal to \pm 1 .

\section{Theoretical Analysis}

The metal spiral is a resonant particle that simultaneously exhibits electric, magnetic, and chiral responses. In the presence of an electromagnetic field, the response of the spiral can be described by the following equations (Serdyukov et al. 2001):

$$
\begin{aligned}
& \mathbf{p}=\varepsilon_{0} \alpha_{e e} \mathbf{E}+i \sqrt{\varepsilon_{0} \mu_{0}} \alpha_{e m} \mathbf{H}, \\
& \mathbf{m}=\alpha_{m m} \mathbf{H}+i \sqrt{\frac{\varepsilon_{0}}{\mu_{0}}} \alpha_{m e} \mathbf{E},
\end{aligned}
$$

where $\mathbf{p}$ and $\mathbf{m}$ are the induced electric- and magnetic-dipole moments, $\mathbf{E}$ and $\mathbf{H}$ are the external electric and magnetic fields, $\alpha_{e e}$ and $\alpha_{m m}$ are tensors of dielectric and magnetic susceptibilities, and $\alpha_{e m}$ and $\alpha_{m e}$ are pseudotensors that characterize the chiral properties of the spiral. From the principle of symmetry of kinetic coefficients, it follows that (Serdyukov et al. 2001)

$$
\alpha_{e m}=\alpha_{m e}^{T}
$$

where $T$ means transposition.

In Semchenko et al. (2007b), it has been proposed that a metal helix can emit a circularly polarized wave under a linearly polarized excitation when the electric field vector $\mathbf{E}$ is parallel to the axis of the helix. For $x$ being the axis of the helix, the projected components of the induced electric $p$ and magnetic $m$ dipole moments can be written as

$$
\begin{aligned}
& p_{x}=\frac{i}{\omega} \int_{x_{1}}^{x_{2}} I(x) d x, \\
& m_{x}=\frac{1}{2} R^{2} q \int_{x_{1}}^{x_{2}} I(x) d x,
\end{aligned}
$$

where $\omega$ is the cyclic frequency of the current in the helix, $I$ is the current strength, $r$ is the helix radius, and $q$ is the relative torsion of a helix, which is related to the 
helix pitch $h$ as

$$
h=\frac{2 \pi}{|q|} .
$$

The sign of the parameter $q$ determines the direction of rotation of the helix in space. If $q>0$, the helix is RH.

The electric and magnetic components are related through the relation

$$
p_{x}=\frac{2 i}{\omega R^{2} q} m_{x} .
$$

This relationship is universal, since it is independent from the current distribution along the helix. The $x$-components of the helix moments play the main role in the radiation of a circularly polarized wave in the direction perpendicular to the helix axis. The universal character of Eq. (7) also applies to an array of helices forming a metamaterial. Evidently, the current flow in a particular helix is induced by both the incident external electromagnetic wave and the field scattered by the neighboring helices. Nevertheless, the $p_{x}$ components of the electric dipole moment and $m_{x}$ of the magnetic moment vary consistently, and Eq. (7) remains valid. Therefore, the geometric parameters of the helix that are presented below ensure radiation of a circularly polarized wave also for a metamaterial consisting of helices.

The generation of a circularly polarized wave by such a helix is possible if

$$
\left|p_{z}\right| \ll\left|p_{x}\right|, \quad \frac{1}{c}\left|m_{z}\right| \ll\left|p_{x}\right|,
$$

where $c$ represents the speed of light. Then, the condition for circularly polarized wave radiation (Semchenko et al., 2007b) reads

$$
\left|p_{x}\right|=\frac{1}{c}\left|m_{x}\right| .
$$

Thus, the components of the electric dipole moment, $p_{y}, p_{z}$, and the magnetic moment $m_{y}, m_{z}$, which are orthogonal to the axis of the helix, should not contribute to the scattered wave. This condition can be achieved in the case of an odd number of turns of the helix. For this purpose, it is necessary to terminate the ends of the spiral in the direction of the incident wave, as shown in Figure 3(a). For this configuration, $p_{z}=0$ and $m_{z}=0$ due to the symmetry of the current distribution along the helix. Although the components $p_{y}$ and $m_{y}$ may not vanish, they do not radiate and, therefore, do not deform the circularly polarized wave.

In order to determine the values of the helix parameters that ensure radiation of a circularly polarized wave, the universal relationship in Eq. (7) and the resonance length condition are used as follows:

$$
\frac{\lambda}{2}=L
$$

where $\lambda$ is the wavelength of the incident electromagnetic wave. By taking into account the relationship between the geometric parameters of the helix,

$$
L \cos \alpha=2 \pi R N_{c},
$$




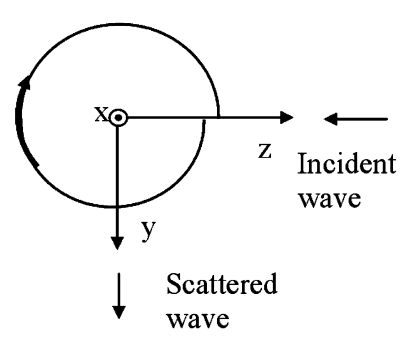

(a)

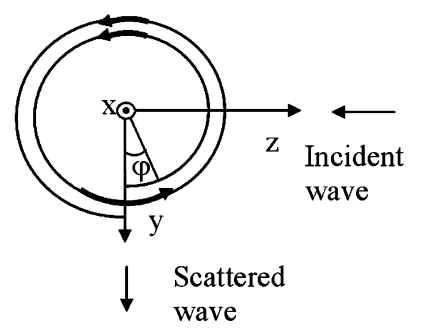

(b)

Figure 3. A schematic representation of the current distribution in: (a) a one-turn helix and (b) a two-turn helix. The arrows indicate the direction of the current flow, and their length is proportional to the intensity of the current. $\varphi$ is the polar angle.

the following trigonometric equation for the helix lead angle $\alpha$ is obtained:

$$
4 N_{c} \tan \alpha=\cos \alpha
$$

or

$$
\sin ^{2} \alpha+4 N_{c} \sin \alpha-1=0
$$

Since angle $\alpha$ takes positive values, the expression for the roots of Eq. (13) can be written in the form

$$
\alpha=\arcsin \left(-2 N_{c}+\sqrt{4 N_{c}^{2}+1}\right) .
$$

The values of the helix lead angle at which helices with different numbers of turns can radiate a circularly polarized wave are listed in Table 1 .

As follows from the table, the radiation of a circularly polarized wave is possible for both odd and even numbers of helix turns. Experimental results show that the intensity of the wave radiated by a helix rapidly decreases as the number of turns increases, i.e., as the helix lead angle decreases. Hence, the optimum number of turns is $N_{c}=1$ or 2. For a single-turn helix, $N_{c}=1$ (Figure 3(a)), and it is necessary to eliminate the influence of the $p_{y}$ and $m_{y}$ components of the electric and magnetic moments that are orthogonal to the helix axis. Therefore, in order to obtain a circularly polarized wave, the ends of a single-turn helix should be directed towards the incident wave. The current distribution along a helix with two turns, $N_{c}=2$ (Figure 3(b)) is more symmetric, and a circularly polarized wave is radiated for any orientation of the helix ends relative to the wavevector.

\section{Table 1}

The optimum values of the helix lead angle $(\alpha)$ that correspond to radiation of a circularly polarized wave for different values of the number of turns $\left(N_{c}\right)$

\begin{tabular}{ccccccccc}
\hline$N_{c}$ & 1 & 2 & 3 & 4 & 5 & 6 & 7 & 8 \\
\hline$\alpha$ (degrees) & 13.7 & 7.1 & 4.7 & 3.6 & 2.9 & 2.4 & 2.0 & 1.8 \\
\hline
\end{tabular}


Based on this formulation, the numerical values of the helix parameters that ensure radiation of a circularly polarized wave when the helix is excited by a linearly polarized wave can be obtained. As an example, the values for the two-turn helix working at $f=3 \mathrm{GHz}$ are calculated. The length of the helix wire should correspond to the condition of the main frequency resonance, i.e., $\lambda / 2, L=50 \mathrm{~mm}$. The helix lead angle that is realized at $N_{c}=2$ is chosen from the table: $\alpha=7.1^{\circ}$. The helix radius can be calculated from Eq. (11): $R=3.95 \mathrm{~mm}$. The helix pitch is found from the relationship $h=\frac{L \sin \alpha}{N_{c}}$ or $h=3.1 \mathrm{~mm}$.

The obtained theoretical results for true spirals were experimentally checked for the lattice of a spiral with calculated optimal parameters (Semchenko et al., 2004, 2007b; Khakhomov et al., 2006; Samofalov \& Tretyakov, 2006). As was noted before, the results for lattice are also valid for single spiral due to the universality of relationship in Eq. (7).

The investigation of canonical spirals is also interesting since such a spiral can be represented as a combination of a wire loop and linear conductors. Taking into account the input impedance of the wire $\left(Z_{W}\right)$ and the loop $\left(Z_{L}\right)$, the resonance occurs when these admittances cancel out each other, i.e., $Y_{W}=-Y_{L}$. After some approximations, explained in detail in Asghar et al. (2007a, 2007b) and Semchenko et al. (2007e), a simplified expression for the optimal dimensions of canonical spirals was obtained:

$$
l \approx k_{0} \pi R^{2} .
$$

Using the resonant condition for the total length of the spiral being close to $\lambda / 2$,

$$
2 \pi R+2 l=\frac{\lambda}{2}
$$

the radius of the spiral in terms of the working frequency reads

$$
R=\frac{\lambda}{4 \pi}(\sqrt{3}-1) .
$$

Therefore, for a given frequency, by applying Eqs. (16) and (17), the main constitutive parameters of the helix $R$ and $l$ can be calculated.

One major advantage of the true and canonical optimal spirals is that their response is independent from the polarization of the incident field (transverse electric [TE] or transverse magnetic [TM]). This is demonstrated in the next section through numerical simulation of the electromagnetic response of helices for different incident-field polarizations.

\section{Modeling of Single Spirals}

In this section, the scattering properties of slabs formed by periodical arrays of single spirals is investigated by numerical simulations (Ansoft-HFSS, Spain). Periodic boundary conditions (PBCs) have been applied in order to simulate a uniform array of helices, i.e., RH or LH helices. Notice that this condition is different from the perfect electrical conductor (PEC) condition imposed on the walls of a regular waveguide, which would imply a mirror symmetry and, thus, a racemically ordered array of helices. Both the canonical spiral and true helix are simulated. For the canonical spiral, the optimum parameters are calculated numerically by taking the input impedance of the wire and loop elements into account. The respective scattering properties are then compared to those obtained using some approximations. 


\subsection{Canonical Spirals}

4.1.1. Optimum Parameters Based on the Input Impedance. In order to analyze the scattering behavior of canonical spirals, the optimum parameters numerically calculated in Asghar et al. (2007b) for a resonant frequency of $8 \mathrm{GHz}$ are considered: $R=1.95 \mathrm{~mm}$, $l=2.85 \mathrm{~mm}$. The reflection and transmission coefficients were calculated with AnsoftHFSS for a TE and TM polarization of the incident electric field. In the TE case, the electric field $(E)$ was polarized along the wire, and the magnetic field $(H)$ was parallel to the loop (see the left panel of Figure 4(a)). The resonance frequency is found to be $7.4 \mathrm{GHz}$, slightly lower than the design. The same behavior is observed for the TM polarization of the incident field, i.e., $H$ field axial to the loop. These results are depicted in Figure 4(b).

In order to check the polarization of the reflected field, the $\hat{y}$ and $\hat{z}$ components of the reflected field were calculated (see the right panels of Figure 4). It is observed that, at a certain point around the resonant frequency, both components have the same modulus. The $90^{\circ}$ phase shift between components required for circular polarization was verified with Ansoft-HFSS.

4.1.2. Approximation of the Parameters. One approximation in modeling the spiral's response is based on the input impedance of the wire and the loop (Eq. (15)). In order to validate this approximation, the same resonance frequency is set $(8 \mathrm{GHz})$, and applying Eqs. (16) and (17), the dimensions of the spirals are calculated: $R=2.18 \mathrm{~mm}$ and $l=2.51 \mathrm{~mm}$. The results of the $S$ parameters and the $\hat{y}$ and $\hat{z}$ components of the
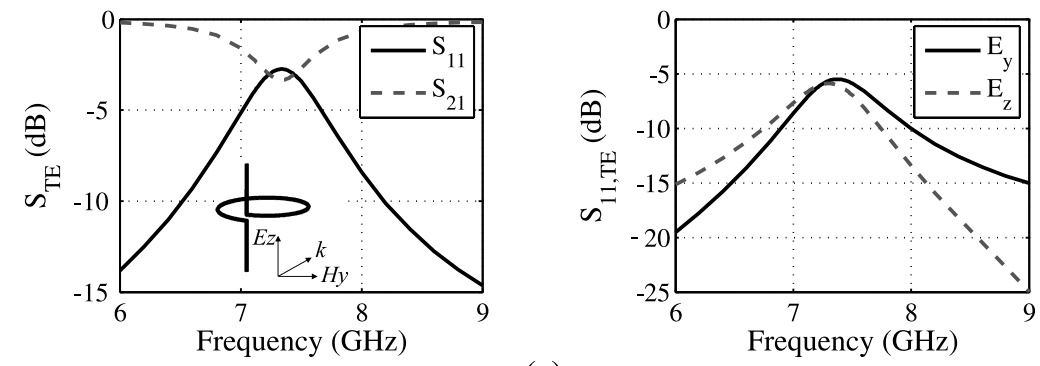

(a)
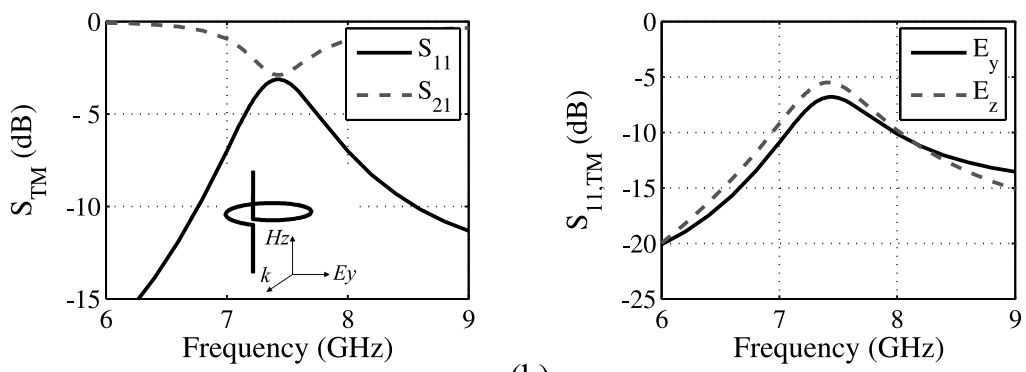

(b)

Figure 4. Canonical spiral with optimum dimensions $R=1.95 \mathrm{~mm}, l=2.85 \mathrm{~mm}, r_{0}=0.1 \mathrm{~mm}$, and $h=0.3 \mathrm{~mm}$. Transmission $\left(S_{21}\right)$ and reflection $\left(S_{11}\right)$ spectra and $\hat{y}$ and $\hat{z}$ components of the reflected field $\left(E_{y}, E_{z}\right)$ for: (a) the TE polarization of the incident electric field and (b) the TM polarization of the incident electric field. The insets represent the polarization of the incident field. 

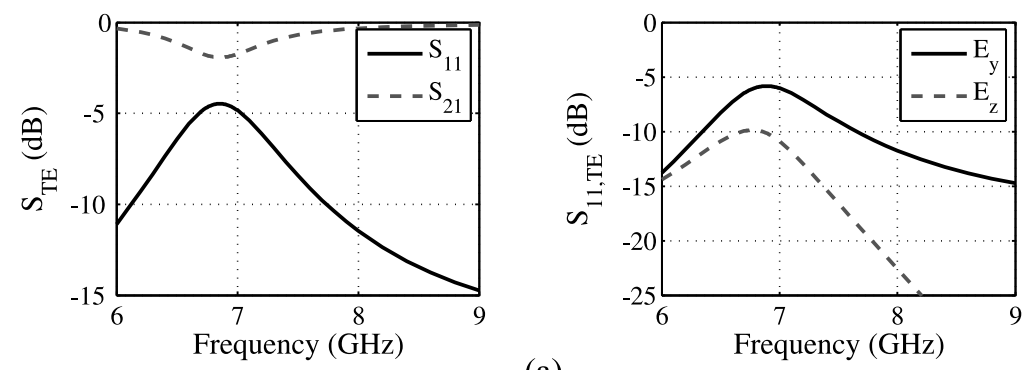

(a)
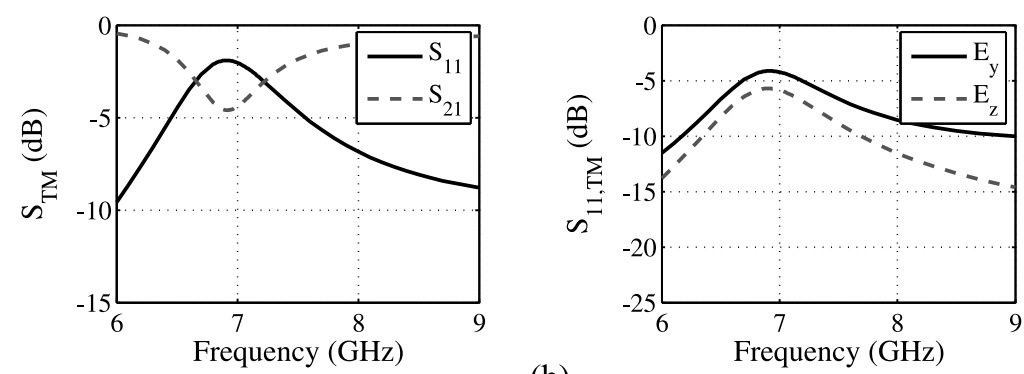

(b)

Figure 5. Canonical spiral with approximated dimensions $R=2.18 \mathrm{~mm}, l=2.51 \mathrm{~mm}, r_{0}=$ $0.1 \mathrm{~mm}$, and $h=0.3 \mathrm{~mm}$. Transmission $\left(S_{21}\right)$ and reflection $\left(S_{11}\right)$ spectra and $\hat{y}$ and $\hat{z}$ components of the reflected field $\left(E_{y}, E_{z}\right)$ for: (a) the TE polarization of the incident electric field and (b) the TM polarization of the incident electric field.

reflected electric field are shown in Figure 5. Compared with the previous results, the resonance frequency is shifted downward, but the same resonance is observed for TE or TM polarization of the incident electric field. The maximum circularity of the reflected field is obtained around the resonance frequency (see right panels in Figure 5); however, the $\hat{y}$ and $\hat{z}$ components of the reflected field are not exactly equal.

4.1.3. Scattering Parameters Versus Density of Inclusions. In this section, the effect of the density of inclusions in a slab on the scattering parameters is analyzed. To do so, the size (width and height) of the "waveguide" (with PBCs), which contains the spiral, is varied. Initially a square "waveguide" with the dimensions $w=5.62 \mathrm{~mm}=0.3 \lambda_{0}$ at $8 \mathrm{GHz}$ is considered, and the parameters $d_{y}$ and $d_{z}$ are varied (see Figure 6). The dimensions of the spiral are $R=2.18 \mathrm{~mm}, l=2.51 \mathrm{~mm}, r_{0}=0.05 \mathrm{~mm}$, and $h=0.2 \mathrm{~mm}$.

The influence of the separation between spirals in the $\hat{y}$ and $\hat{z}$ directions has been analyzed by fixing the parameter $d_{z}$ and varying $d_{y}$ and vice versa. The results are depicted in Figure 7. It is observed that for a fixed $d_{z}$, as the lateral distance between spirals $d_{y}$ increases (direction of the arrows in Figure 7(a)), the resonance frequency and the strength of the resonance do not change. The decrease in the bandwidth is the only noticeable effect. For a fixed $d_{y}$ (Figure 7(b)), as $d_{z}$ increases (direction of the arrows), the resonance frequency increases, and the bandwidth decreases. This is reasonable because $d_{z}$ defines the gap between different layers of wires, i.e., it affects the capacitance. Assuming an $L C$ circuit formed by the wire and the gap, if $C$ decreases, the resonant frequency increases. 


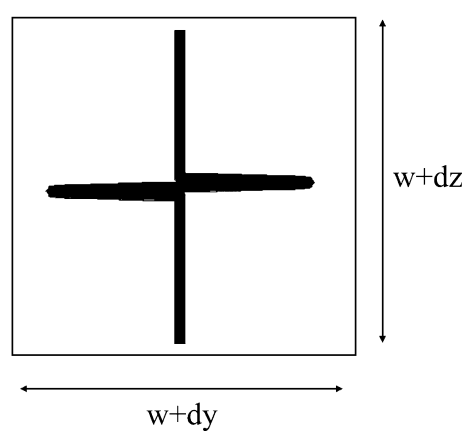

Figure 6. Canonical spiral inside a "waveguide" with PBCs. Initial lattice constant $w=5.62$ $\mathrm{mm}=0.3 \lambda_{0}$ at $8 \mathrm{GHz}$.

\subsection{Single-Turn True Spirals}

In this section, the scattering parameters are investigated for true spirals (see Figure 2). Following the design formulas presented in the theoretical analysis, the optimum parameters of the helix for a resonance frequency of $3 \mathrm{GHz}$ are: $N_{c}=1, R=7.75 \mathrm{~mm}$, $\alpha=13.6^{\circ}, L=50 \mathrm{~mm}, h=12 \mathrm{~mm}$, and $2 r_{0}=1.55 \mathrm{~mm}$. Initially, the true helix is located in a square "waveguide" with the dimensions $w_{y}=w_{z}=25.83 \mathrm{~mm}=0.258 \lambda$ at $3 \mathrm{GHz}$, and the scattering for a TE and TM polarization of the electric field have been calculated. In the case of TE polarization, the electric field is axial to the spirals $\left(E_{z}, H_{y}\right)$ and for the TM, the magnetic field is the axial one $\left(E_{y}, H_{z}\right)$. The $S$ parameters and the $\hat{y}$ and $\hat{z}$ components of the reflected field for both polarizations are shown in Figure 8 . For TE and TM polarizations, the resonant frequency is at $3 \mathrm{GHz}-$ exactly at the design frequency. At this frequency, the $\hat{y}$ and $\hat{z}$ components of the reflected field have the same magnitude, showing a $90^{\circ}$ phase shift between them, which has been checked with HFSS.

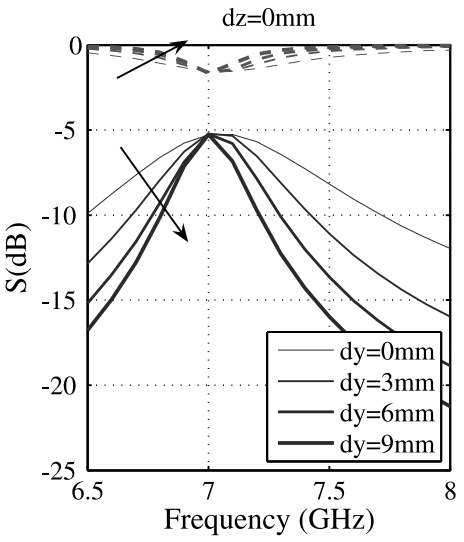

(a)

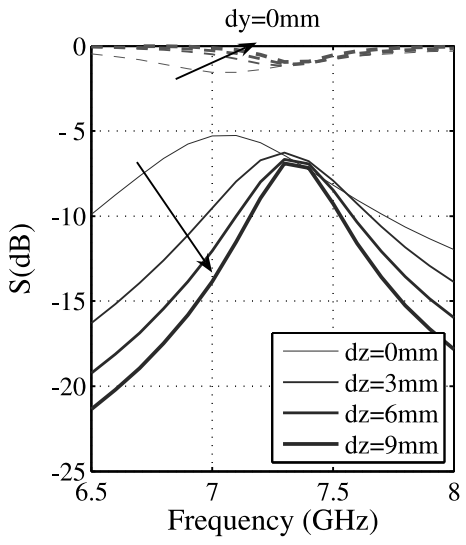

(b)

Figure 7. Canonical spiral with approximated dimensions and scattering parameters vs. distance in: (a) the $\hat{y}$ direction and (b) the $\hat{z}$ direction. The continuous line represents the reflection coefficient $\left(S_{11}\right)$, and the dashed line presents the transmission coefficient $\left(S_{21}\right)$. The arrows indicate the trend of the curves as the parameter increases. 

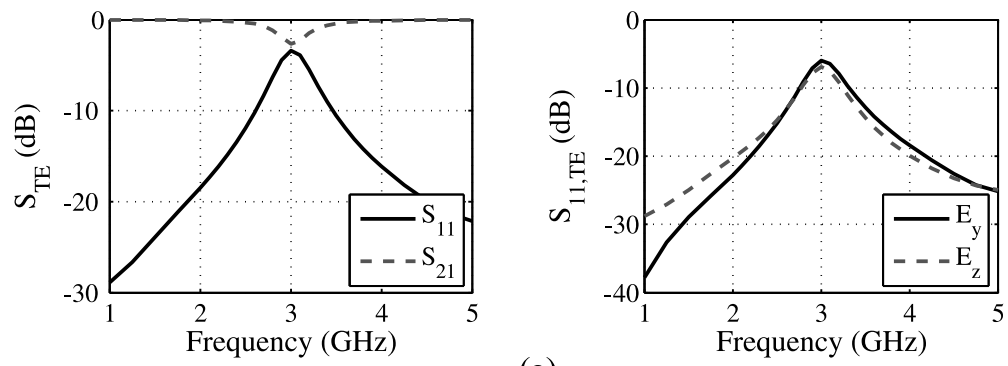

(a)
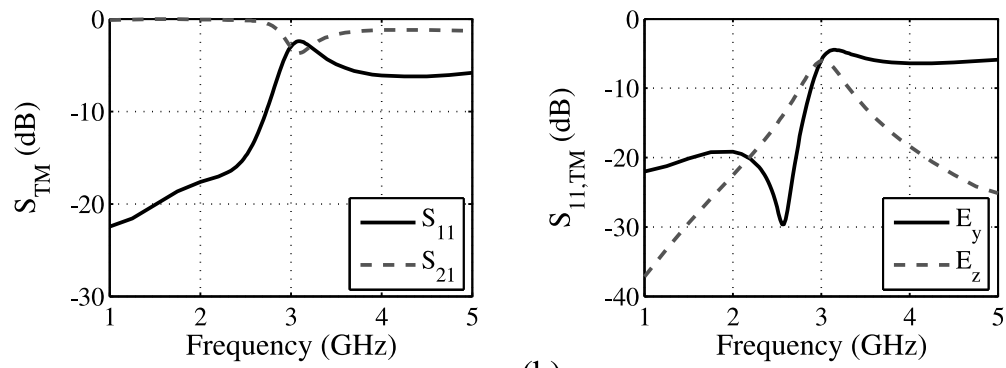

(b)

Figure 8. True spiral with transmission $\left(S_{21}\right)$ and reflection $\left(S_{11}\right)$ spectra and $\hat{y}$ and $\hat{z}$ components of the reflected field $\left(E_{y}, E_{z}\right)$ for: (a) the TE polarization of the incident electric field and (b) the TM polarization of the incident electric field.

Therefore, the reflected field is circularly polarized. Out of the resonant frequency, the behavior of the reflected field differs slightly for both polarizations.

4.2.1. Scattering Parameters Versus Density of Inclusions. Analogous to the canonical case, the influence of the helix concentration was analyzed by independently varying $d_{z}$ and $d_{y}$. The results are depicted in Figure 9. It is observed that for both variations $d_{y}$ and $d_{z}$, the density of inclusions does not affect the resonant frequency of the slab, increasing the reflection bandwidth as the concentration increases. Compared to the canonical spirals, since there are no wires, no capacitance occurs between the helices, and therefore, the resonant frequency does not change with $d_{z}$.

\subsection{Two-Turn True Spirals}

The stability of the circularly polarized reflected wave of a two-turn helix with respect to the angle of incidence of the incident field was analyzed. As it was explained in the theoretical part, the current distribution in a two-turn helix is more symmetrical than in the one-turn case, and the radiation of circularly polarized wave takes place for any orientation of the ends of the helix relatively to the wave vector of the incident wave. Three cases were considered: the end of the helix is rotated $90^{\circ}$ (i.e., perpendicular to the incident field), $45^{\circ}$, and $0^{\circ}$ (i.e., oriented toward the incident field). The parameters of the helix are the ones calculated in the theoretical part: $N_{c}=2, R=3.95 \mathrm{~mm}, \alpha=7.1^{\circ}$, $L=50 \mathrm{~mm}, h=3.1 \mathrm{~mm}$, and $2 r_{0}=0.4 \mathrm{~mm}$.

The transmission and reflection spectra and the $\hat{y}$ and $\hat{z}$ components of the reflected field for the three positions of the helix are depicted in Figure 10 for TE- or TM-polarized 


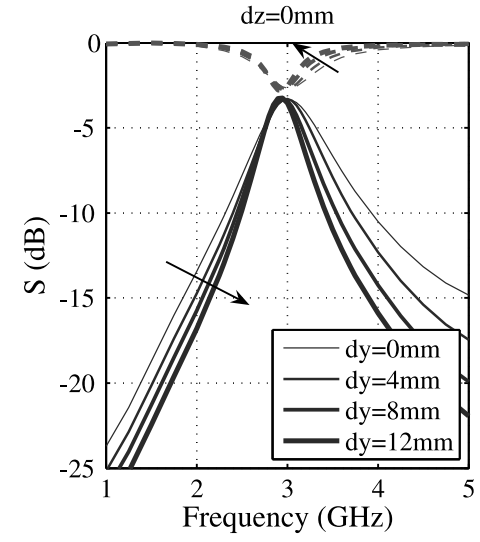

(a)

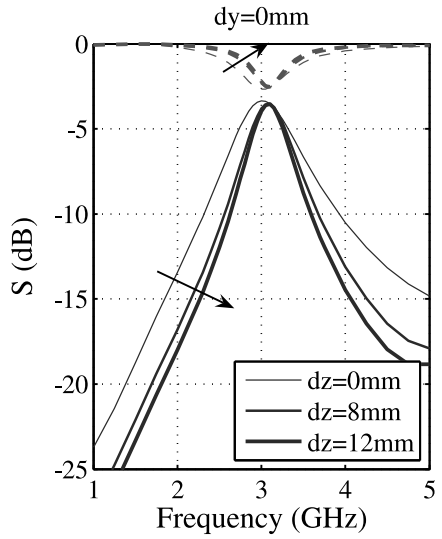

(b)

Figure 9. True spiral with scattering parameters vs. distance in: (a) the $\hat{y}$ direction and (b) the $\hat{z}$ direction. $S_{11}$ is shown by the continuous line; $S_{21}$ corresponds to the dashed line. The arrows indicate the trend of the curves as the parameter increases.
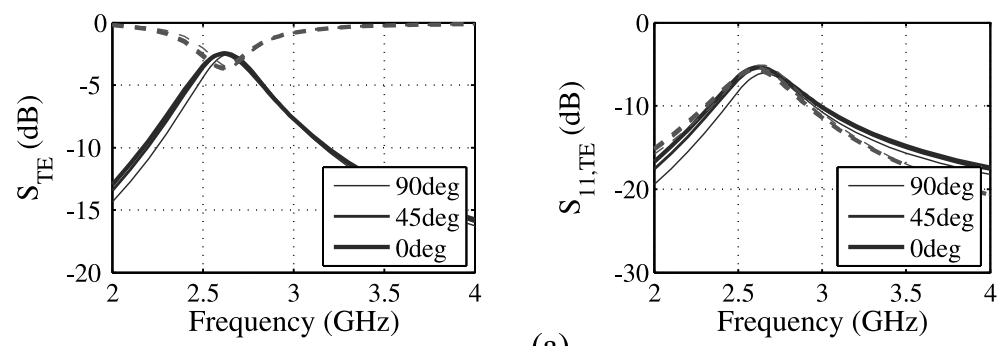

(a)
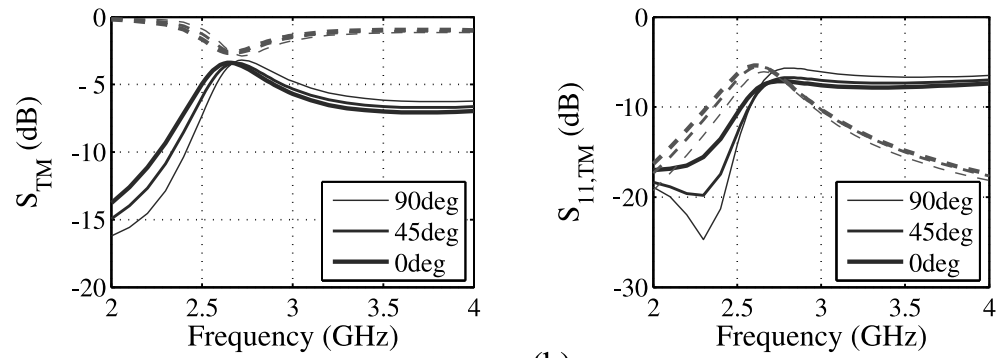

(b)

Figure 10. Two-turn helix with transmission $\left(S_{21}\right)$ and reflection $\left(S_{11}\right)$ spectra and $\hat{y}$ and $\hat{z}$ components of the reflected field $\left(E_{y}, E_{z}\right)$ for an angle of the incident field with respect to the end of the helix of $90^{\circ}, 45^{\circ}$, and $0^{\circ}$ : (a) TE polarization and (b) TM polarization. 
incident fields. In both cases, it is observed that the resonant frequency does not change with the angle of incidence. Moreover, the frequency of circular polarization coincides with the resonant frequency and does not depend on the angle of incidence.

\section{Modeling Racemic Pairs of Spirals}

In this section, we study and present compensation of chirality in racemic mixtures of spirals, i.e., mixtures with an equal number of RH and LH helices per unit cell. The effect of the density of inclusion pairs and the distance between helices in the pair is analyzed.

\subsection{RH-LH Canonical Spirals}

The geometry of the RH-LH pair of canonical spirals is shown in Figure 11. Both spirals are immersed in a "waveguide" and, as in the previous analysis, PBCs are applied. The initial dimensions of the waveguide are $w_{y}=9.6 \mathrm{~mm}$ and $h_{z}=6.5 \mathrm{~mm}$, and the distance between the spiral's axis is $d=4.5 \mathrm{~mm}$. The lateral distance between pairs is defined by $w_{y}+d_{y}$, the perpendicular distance between parallel layers of pairs is $h_{z}+d_{z}$, and the distance between helices is $d+d_{\text {helix }}$. The dimensions of the spirals are the ones calculated numerically in Section 4.1.1: $R=1.95 \mathrm{~mm}, l=2.85 \mathrm{~mm}, r_{0}=0.1 \mathrm{~mm}$, and $h=0.3 \mathrm{~mm}$.

The scattering properties of the initial case, i.e., $d_{y}=d_{z}=d_{\text {helix }}=0$, have been calculated for a TE and TM incident field, as shown in Figure 12. The resonance frequency is found to be $7.3 \mathrm{GHz}$ for both polarizations. Due to the mixing of $\mathrm{RH}$ and LH spirals, compensation of chirality is achieved, and therefore, an enhancement of the resonance is observed with respect to the case of single spirals: $S_{11}=-0.063 \mathrm{~dB}$ and $S_{21}=-24.02 \mathrm{~dB}$ at $7.3 \mathrm{GHz}$. As a consequence of chirality compensation, the reflected wave is linearly polarized in the direction of the incident field instead of being circularly polarized, as it was for single spirals. This can be observed by looking at the components of the reflected field (the right panel of Figure 12). For the TEpolarized incident field $\left(E_{z}\right)$, the reflected wave at the resonant frequency is also along $\hat{z}\left(E_{z}=0 \mathrm{~dB}, E_{y}=-30 \mathrm{~dB}\right)$, but for TM incident fields $\left(E_{y}\right)$, the reflected wave is linearly polarized along $\hat{y}\left(E_{y}=0 \mathrm{~dB}, E_{z}=-35 \mathrm{~dB}\right)$.

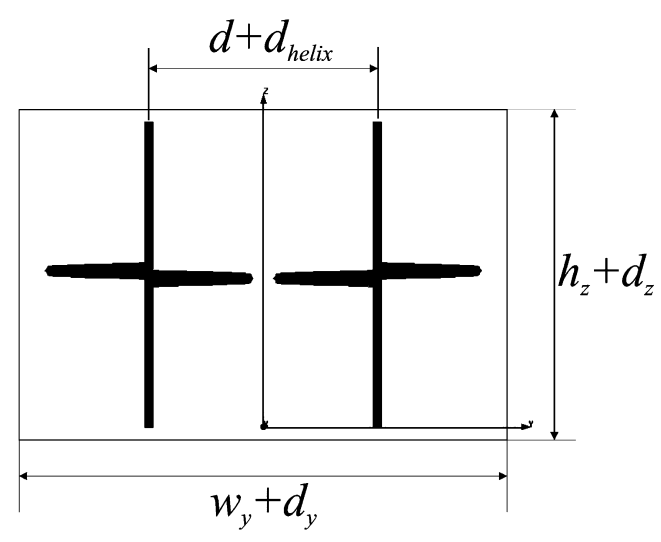

Figure 11. Geometry of the RH-LH pair of canonical spirals. 

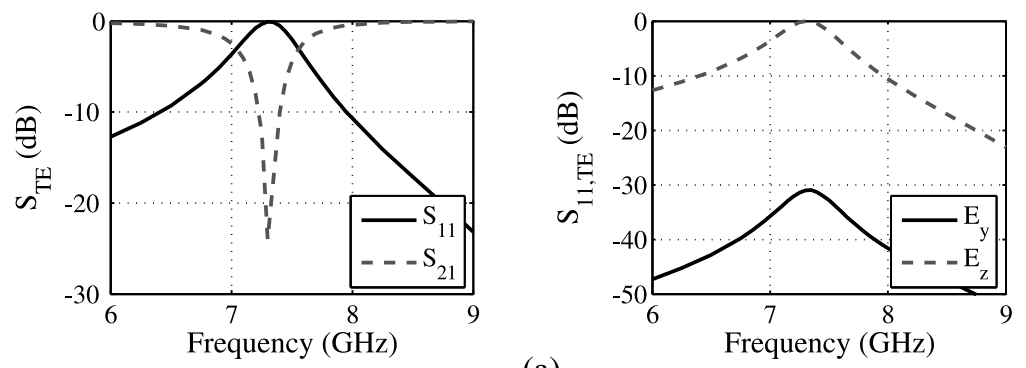

(a)
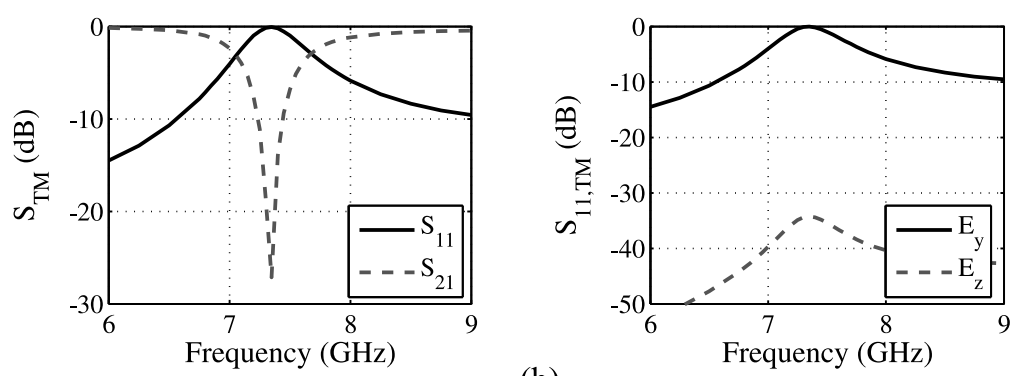

(b)

Figure 12. RH-LH canonical spirals with transmission $\left(S_{21}\right)$ and reflection $\left(S_{11}\right)$ spectra and $\hat{y}$ and $\hat{z}$ components of the reflected field $\left(E_{y}, E_{z}\right)$ for: (a) TE polarization of the incident electric field and (b) TM polarization of the incident electric field.

5.1.1. Scattering Parameters Versus Density of Pairs As in the previous cases, the concentration of RH-LH helices was modified by varying the lateral and perpendicular distances between pairs. In order to study the influence of the lateral distance between pairs, the parameters $d_{z}$ and $d_{\text {helices }}$ were fixed to zero, and $d_{y}$ was varied. The results are depicted in Figure 13(a). It is observed that as the distance between pairs increases, the resonant frequency slightly decreases and the reflection bandwidth also decreases. This effect is stronger in the upper part of the frequency band.

The influence of the separation in the $z$ direction was also analyzed (see Figure 13(b)). For a fixed $d_{y}$ and $d_{\text {helices }}$, as $d_{z}$ increases, the resonant frequency increases. Notice that this effect is not linear; for $d_{z}$ equal to 0,3 , and $6 \mathrm{~mm}$, the resonant frequencies are 7.3, 7.55, and $7.6 \mathrm{GHz}$, respectively. The reflection bandwidth decreases, and this effect is stronger in the lower part of the bandwidth.

5.1.2. Distance Between Spirals of the Pair. The influence of the distance between the RH and LH spirals has been studied (see Figure 14). According to these results, this separation mainly affects the bandwidth (as the distance increases, the bandwidth decreases) rather than the resonant frequency. This result is consistent with the one obtained for single spirals (see Figure 7), where it was observed that the resonant frequency does not depend on the lateral distance between inclusions.

\subsection{RH-LH One-Turn True Spirals}

Analogously, RH-LH pairs of true spirals (see Figure 15) were analyzed. The initial dimensions of the waveguide are $w_{y}=h_{z}=36.1 \mathrm{~mm}$, and the distance between the 


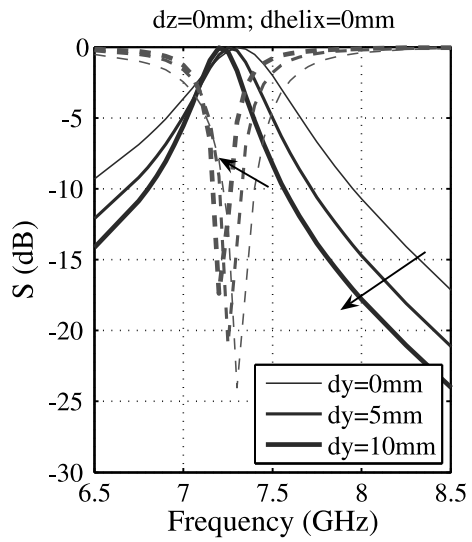

(a)

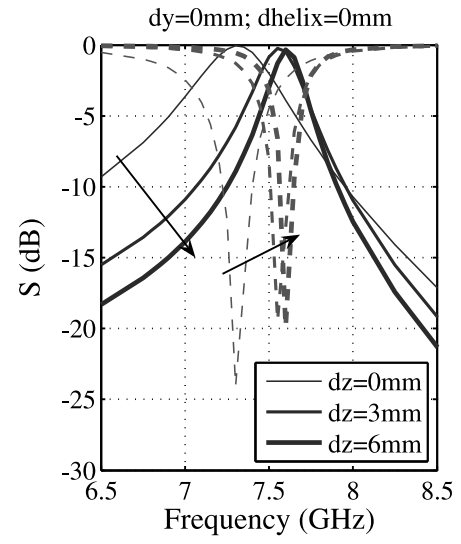

(b)

Figure 13. RH-LH canonical spirals with Scattering parameters vs. distance in (a) the $\hat{y}$ direction and (b) the $\hat{z}$ direction. $S_{11}$ is shown by the continuous line, and $S_{21}$ corresponds to the dashed line. The arrows indicate the trend of the curves as the parameter increases.

centers of spirals $d=18 \mathrm{~mm}$. The dimensions of one-turn helices are the same as in the case of single true spirals: $f=3 \mathrm{GHz}, R=7.75 \mathrm{~mm}, \alpha=13.6^{\circ}, L=50 \mathrm{~mm}$, $h=12 \mathrm{~mm}$, and $2 r_{0}=1.55 \mathrm{~mm}$.

The scattering parameters for the smallest lattice constant, i.e., $d_{y}=d_{z}=d_{\text {helix }}=0$, are shown in Figure 16(a) and 16(b) for TE and TM polarizations of the incident electric field, respectively. Due to chirality compensation, when the spirals are excited with a TE incident wave ( $E$ field along $\hat{z}$ direction), the resonant frequency is at $2.86 \mathrm{GHz}$ and the reflected field is linearly polarized along $\hat{z}$. However, in the case of a TM incident field ( $E$ field along $\hat{y}$ ), the resonant frequency is at $2.9 \mathrm{GHz}$ and the reflected field is linearly polarized along the $\hat{y}$ axis.

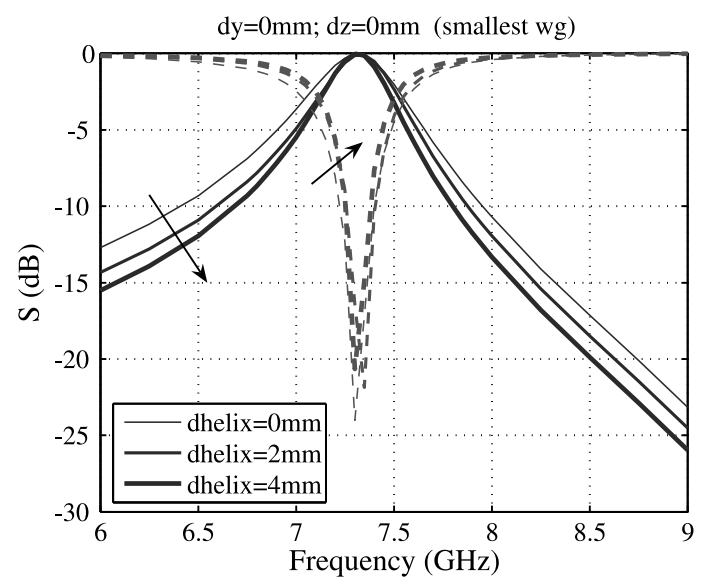

Figure 14. RH-LH canonical spirals with scattering parameters vs. distance in the $\hat{z}$ direction for a TE-polarized incident plane wave. The arrows indicate the trend of the curves as the parameter increases. 


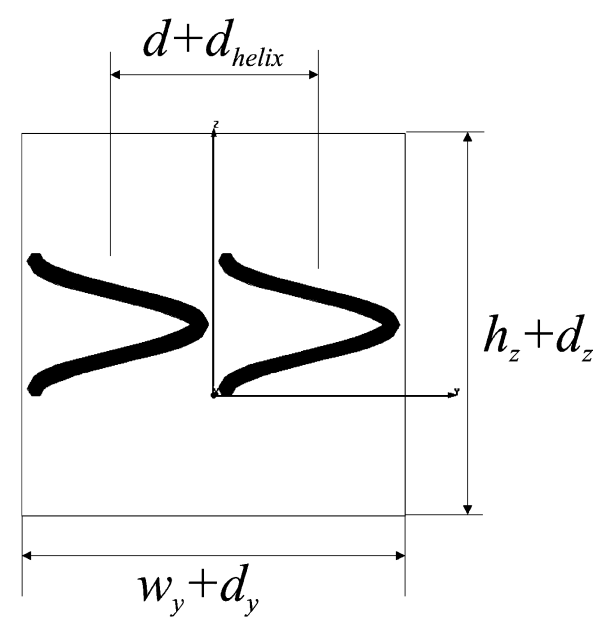

Figure 15. Geometry of the RH-LH pair of canonical spirals.

5.2.1. Scattering Parameters Versus Density of Pairs. The scattering parameters versus density of pairs were analyzed, and the results are shown in Figure 17. As the distance between pairs increases (Figure 17(a)), the resonant frequency slightly decreases (notice the small frequency band that is plotted). The $S_{11}$ bandwidth also decreases, and this effect is stronger in the upper part of the band. The perpendicular distance between spirals
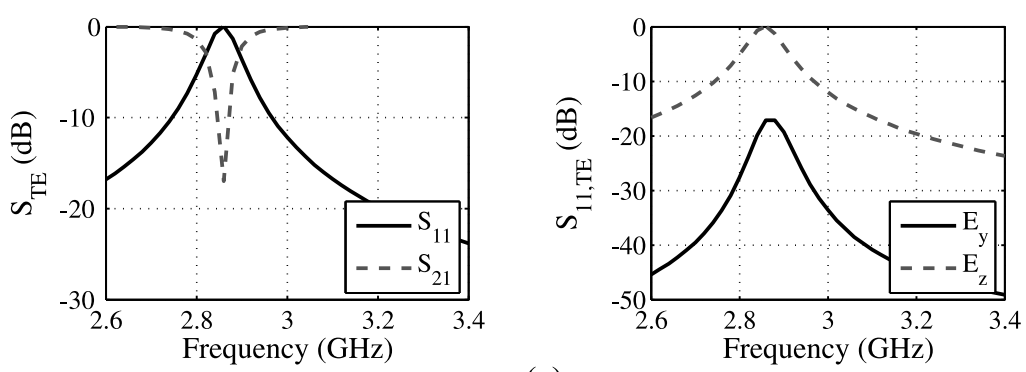

(a)
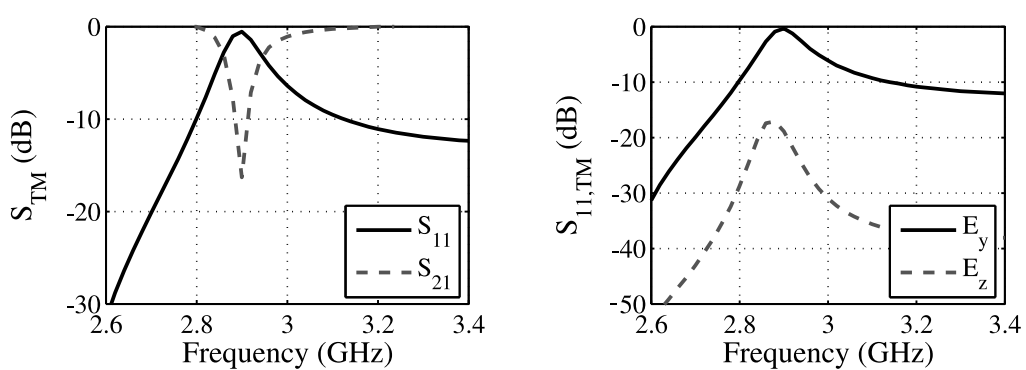

(b)

Figure 16. RH-LH true spirals with transmission $\left(S_{21}\right)$ and reflection $\left(S_{11}\right)$ spectra and $\hat{y}$ and $\hat{z}$ components of the reflected field $\left(E_{y}, E_{z}\right)$ for: (a) TE polarization of the incident electric field and (b) TM polarization of the incident electric field. 


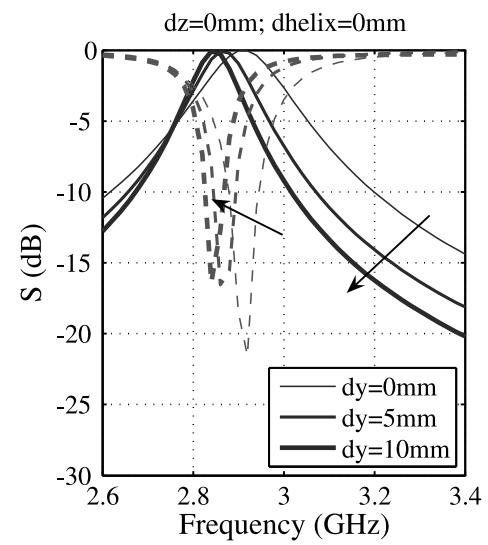

(a)

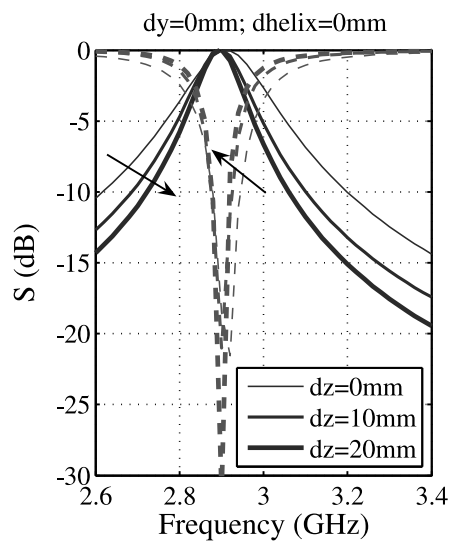

(b)

Figure 17. RH-LH true spirals with scattering parameters vs. density of pairs: (a) lateral distance in the $\hat{y}$ direction and (b) perpendicular distance in the $\hat{z}$ direction. $S_{11}$ is shown by the continuous line; $S_{21}$ corresponds to the dashed line. The arrows indicate the trend of the curves as the parameter increases.

(Figure 17(b)) does not change the resonant frequency but only affects the bandwidth. These results agree with the ones obtained in the analysis of single true spirals.

5.2.2. Distance Between Spirals. The influence of the distance between the RH and LH spirals was also studied (see Figure 18). As the distance between spiral increases, the resonant frequency slightly decreases. The bandwidth is also reduced, mainly in the upper part of the window.

5.2.3. Very Low Density of Pairs. Finally, the scattering parameters for a very low density of pairs $\left(d_{y}, d_{z}=40 \mathrm{~mm}\right.$ ) were calculated (see Figure 19). Comparing to

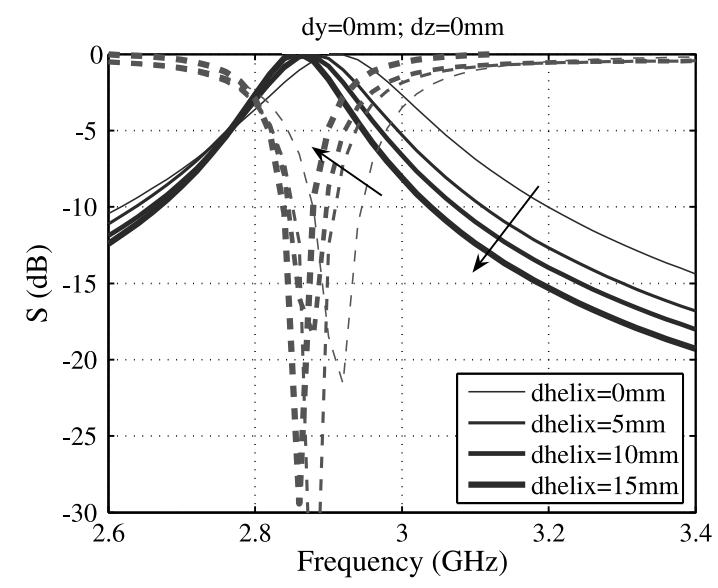

Figure 18. RH-LH true spirals with scattering parameters vs. distance in the $\hat{z}$ direction. The arrows indicate the trend of the curves as the parameter increases. 


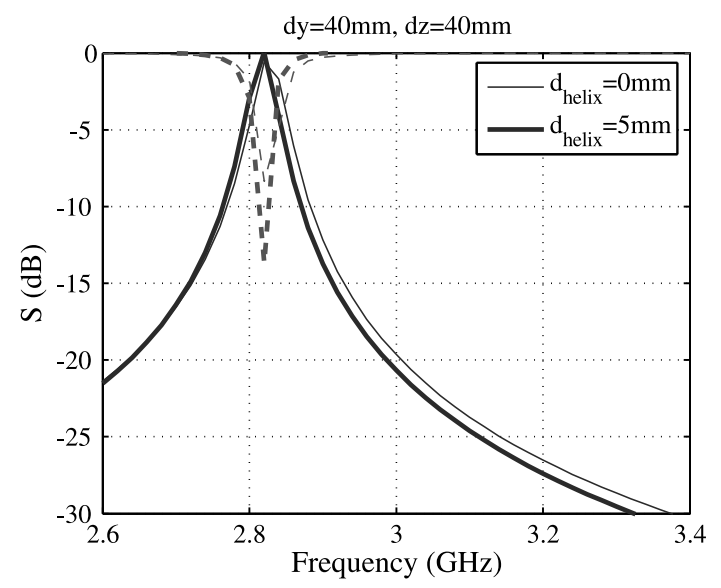

Figure 19. RH-LH true spirals with scattering parameters for a very large distance between pairs $\left(d_{y}, d_{z}=40 \mathrm{~mm}\right)$.

Figure 18, it is observed that as the distance between pairs gets close to $\lambda$, the resonance gets weaker and narrower but is still noticeable and stable in the frequency; i.e., there is no frequency shift with respect to the case of higher density of inclusions.

\section{Conclusions}

In this article, theoretical analysis and modeling of spirals with optimal parameters have been presented. It has been observed that for the optimal parameters of the spirals, it is possible to obtain equal dielectric, magnetic, and chiral susceptibilities. Two different types of spirals have been investigated: canonical helices and single- or two-turn true helices.

The reflection and transmission coefficients of spirals under plane wave excitation have been calculated with Ansoft-HFSS. It has been observed that when single spirals ( $\mathrm{RH}$ or LH) are arranged in an array configuration, the reflected wave is circularly polarized around the resonant frequency. However, when RH-LH pairs of helices are placed together, compensation of the chirality is achieved, and the reflected wave is linearly polarized. It has been proven that the density of inclusions does not affect the resonant frequency of the slab, and only a bandwidth reduction is observed as the density decreases.

\section{Acknowledgment}

The research presented in this article has been financially supported by METAMORPHOSE NoE funded by the E.C. under contract NMP3-CT-2004-50252.

\section{References}

Asghar, M., I. Hakala, J. Jantunen, H. Kettunen, M. Pitkonen, J. Qi, G. Statkute, A. Varpula, I. V. Semchenko, S. A. Khakhomov, R. Gonzalo, E. Ozbay, V. Podlozny, A. Sihvola, S. Tretyakov, \& H. Wallen. 2007a. Electromagnetic cloaking with a mixture of spiral inclusions. Proc. Metamaterials 2007, Rome, Italy, 22-26 October, 957-960. 
Asghar, M., I. Hakala, J. Jantunen, H. Kettunen, J. Qi, \& A. Varpula. 2007b. Electromagnetic cloaking with a mixture of spiral inclusions. Metamaterials in Electromagnetics and Radio Engineering, Helsinki University of Technology. On-line course, available at: http://metamorphosevi.org/

Bohren, C. F., R. Luebers, H. S. Langdon, \& F. Hunsberger. 1992. Microwave-absorbing chiral composites: Is chirality essential or accidental. Appl. Optics 31:6403-6407.

Cloete, J. H., M. Bingle, \& D. B. Davidson. 1999. The role of chirality in synthetic microwave absorbers. Proceedings of the International Conference on Electromagnetics in Advanced Applications, Torino, Italy, September, 55-58.

Khakhomov, S. A., I. V. Semchenko, A. L. Samofalov, \& S. A. Tretyakov. 2006. Obtaining circularly polarized reflected electromagnetic waves by the artificial flat lattice with one-turn helies. Bi-anisotropics 2006, International Conference on Complex Media and Metamaterials, Samarkand, Uzbekistan, 25-27 September, 24-25.

Priou, A., A. Sihvola, S. Tretyakov, \& A. Vinogradov. 1997. Advances in complex electromagnetic materials, NATO ASI Series 3. Dordrecht, The Netherlands: Kluwer Academic Publishers.

Samofalov, A. L., \& S. A. Tretyakov. 2006. Obtaining circularly polarized reflected electromagnetic waves by the artificial flat lattice with one-turn helices. Proceedings of the Gomel State University 39:87-90 (in Russian).

Semchenko, I. V., S. A. Khakhomov, \& M. A. Podalov. 2007a. Device for transformation of polarization of electromagnetics waves. Patent of Belarus, No. 3783, BY 3783 U 2007.08.30.

Semchenko, I. V., S. A. Khakhomov, \& A. L. Samofalov. 2004. Radiation of circularly polarized electromagnetic waves by the artificial flat lattice with two-turns helical elements. Bianasotropics' 2004, 10th International Conference on Complex Media and Metamaterials, Het Pand, Ghent, Belgium, 22-24 September, 236-239.

Semchenko, I. V., S. A. Khakhomov, \& A. L. Samofalov. 2007b. Transformation of the polarization of electromagnetic waves by helical radiators. J. Commun. Technol. Electron. 52:850-855.

Semchenko, I. V., S. A. Khakhomov, \& A. L. Samofalov. 2007c. Device for transformation of polarization of electromagnetics waves. Application to invention of Russian Federation, No. 2006112520, 14.04.2006 (positive decision on patent from 07.06.2007).

Semchenko, I. V., S. A. Khakhomov, \& A. L. Samofalov. 2007d. Device for transformation of polarization. Patent of Belarus, No. 9850, BY 9850 C1 2007.10.30.

Semchenko, I. V., S. A. Khakhomov, \& S. A. Tretyakov. 2007e. Chiral metamaterials with unit negative refraction index. Proc. Metamaterials 2007, Rome, Italy, 22-26 October, 218-221.

Semchenko, I. V., S. A. Khakhomov, S. A. Tretyakov, A. H. Sihvola, \& E. A. Fedosenko. 1998. Reflection and transmission by a uniaxially bi-anisotropic slab under normal incidence of plane waves. J. Phys. D Appl. Phys. 31:2458-2464.

Serdyukov, A. N., I. V. Semchenko, S. A. Tretyakov, \& A. H. Sihvola. 2001. Electromagnetics of bi-anisotropic materials. Amsterdam: Gordon and Breach Science Publishers.

Tretyakov, S. A. 2003. Analytical modeling in applied electromagnetics, Norwood, MA: Artech House.

Tretyakov, S. A., I. S. Nefedov, C. R. Simovski, \& S. I. Maslovski. 2002. Modelling and microwave properties of artificial materials with negative parameters. In Advances in electromagnetics of complex media and metamaterials, 99-122, NATO Science Series II, Vol. 89, Dordrecht: Kluwer Academic Publishers.

Tretyakov, S. A., \& A. A. Sochava. 1993. Proposed composite material for nonreflecting shields and antenna radomes. Electron. Lett. 29:1048-1049.

Tretyakov, S. A., A. A. Sochava, \& C. R. Simovski. 1996. Influence of chiral shapes of individual inclusions on the absorption in chiral composite coatings. Electromagnetics 16:113-127.

Whites, K. W., \& C. Y. Chang. 1997. Composite uniaxial bianisotropic chiral materials characterization: Comparison of predicted and measured scattering. J. Electromag. Waves Appl. 11:371-394.

Zoudi, S., A. Sihvola, \& M. Arsalane. 2002. Advances in electromagnetics of complex media and metamaterials, NATO Science Series II, Vol. 89. Dordrecht: Kluwer Academic Publishers. 